Pontifícia Universidade Catálica $_{\text {Do Rio DE Jamelro }}$

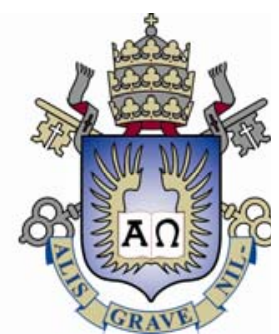

Walter Aliaga Aliaga

\title{
Caracterização do Canal de Propagação GPS em Ambientes Urbanos
}

Dissertação de Mestrado

Dissertação apresentada como requisito parcial para obtenção do título de Mestre pelo Programa de PósGraduação em Engenharia Elétrica da PUC-Rio.

Orientador: Emanoel Paiva de Oliveira Costa

Rio de Janeiro

Novembro de 2010 


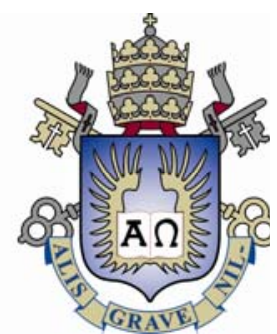

Walter Aliaga Aliaga

\title{
Caracterização do Canal de Propagação GPS em \\ Ambientes Urbanos
}

\begin{abstract}
Dissertação apresentada como requisito parcial para obtenção do título de Mestre pelo Programa de PósGraduação em Engenharia Elétrica da PUC-Rio. Aprovada pela Comissão Examinadora abaixo assinada.
\end{abstract}

Prof. Emanoel Paiva de Oliveira Costa CETUC-PUC-Rio

Prof. Luiz Alencar Reis da Silva Mello CETUC-PUC-Rio

Prof. Marco Antonio Grivet Mattoso Maia CETUC-PUC-Rio

Prof. Rodolfo Sabóia Lima de Souza INMETRO

Prof. José Eugenio Leal Coordenador Setorial do Centro

Técnico Científico - PUC-Rio

Rio de Janeiro, 19 de agosto de 2010 
Todos os direitos reservados. É proibida a reprodução total ou parcial do trabalho sem autorização da universidade, do autor e do orientador.

Walter Aliaga Aliaga

Graduou-se no ano de 2006 em Engenharia Eletrônica pela Pontifícia Universidade Católica do Perú (PUCP)

Ficha Catalográfica

Aliaga Aliaga, Walter
Caracterização do canal de propagação GPS em
ambientes urbanos / Walter Aliaga Aliaga ; orientador:
Emanoel Costa. - 2010.
129 f. : il. (color.) ; $30 \mathrm{~cm}$
Dissertação (mestrado)-Pontifícia Universidade
Católica do Rio de Janeiro, Departamento de Engenharia
Elétrica, 2010.
Inclui bibliografia
1. Engenharia elétrica - Teses. 2. Caracterização do
canal de propagação. 3. GPS. 4. Ambientes urbanos. 5.
Federal Communications Commission. I. Costa, Emanoel.
II. Pontifícia Universidade Católica do Rio de Janeiro.
Departamento de Engenharia Elétrica. III. Título.




\section{Agradecimentos}

Ao Professor Emanoel Costa pela orientação oferecida durante a realização da Dissertação.

Ao Professor Glaucio Lima Siqueira pela orientação oferecida ao começo do Mestrado.

Aos colegas dos Laboratórios 3 e 4 de Radiopropagação do CETUC

Aos amigos do CETUC: Willyan, Bruno, Carlos, Franklin, Harry, Uwe, Alex, Américo, Marcio, Tiago e Juliana.

Aos amigos da casa: Edmundo, Vanessa, Ana Paula, Max, Ronald e Miriam

Ao CAPES pelo financiamento dos estudos de Mestrado

Ao Brasil. 


\section{Resumo}

Aliaga, Walter; Costa, Emanoel. Caracterização do Canal de Propagação GPS em Ambientes Urbanos. Rio de Janeiro, 2010. 129p. Dissertação de Mestrado - Departamento de Engenharia Elétrica, Pontifícia Universidade Católica do Rio de Janeiro.

A presente dissertação tem como tema de estudo a caracterização do canal do Global Positioning System (GPS) para ambientes urbanos. O objetivo do presente estudo é determinar a influência de diversos tipos de ambiente urbano no erro de posição horizontal do receptor GPS, com a finalidade de corroborar se, sob estas condições, o sistema oferece um serviço de posição de acordo com os requerimentos da Federal Communications Commission.Apresentam-se nos diferentes capítulos da dissertação os conceitos básicos do GPS, os procedimentos utilizados na campanha de medidas, as características mais importantes dos ambientes nos quais as mesmas se realizaram e os resultados obtidos, assim como suas análises. Além disso, apresentam-se as configurações dos ambientes utilizados nas simulações, os cálculos para determinar a relação entre azimute e elevação para cada um dos tipos de ambiente adotados, os resultados das simulações e as análises das mesmas. Finalmente, apresentam-se as conclusões para os resultados obtidos durante a campanha de medidas e as simulações, assim como indicações para estudos futuros.

\section{Palavras-chave}

Caracterização do Canal de Propagação; GPS; Ambientes Urbanos; Federal Communications Commission. 


\section{Abstract}

Aliaga, Walter; Costa, Emanoel (Advisor).Characterization of Channel Propagation GPS in Urban Environments. Rio de Janeiro, 2010. 129p. MSc. Dissertation - Departamento de Engenharia Elétrica, Pontifícia Universidade Católica do Rio de Janeiro.

The present dissertation analyzes the propagation channel of the Global Positioning System (GPS) for urban environments. The objective of the study is to determine the influence of different types of urban environments in the error of the horizontal position provided by the GPS receiver. In particular, it investigates if, under degraded conditions, the system is still able to offer a positioning service in accordance to the requirements of the Federal Communications Commission (FCC). The different chapters of the dissertation present the basic GPS concepts, the adopted procedures to perform measurements, as well as the most relevant characteristics of the environments where measurements took place. In continuation, results are described, followed by their analysis. The configurations of environments used in the simulations are depicted, as well as the computations to determine the relationship between azimuth and elevation for each type of environment. Results of such computations and their corresponding analyses are presented for each simulation case. Finally, conclusions on the results obtained from the measurement campaigns and simulations, as well as suggestions for future studies are presented.

\section{Keywords}

Characterization of Channel Propagation; GPS; Urban Environment Federal Communications Commission. 


\section{Sumário}

1. Introdução.

2. Introdução ao Sistema GPS. 16

2.1. Características do Sistema GPS. 17

2.2. Segmentos do GPS. 17

2.3. GPS: A idéia básica. 19

2.4. Estrutura do sinal GPS. 20

2.5. Pseudodistância e cálculo de posição. 21

2.6. Cálculo da posição do usuário. 24

3. Análise de Resultados Numéricos das Medidas. 27

3.1. Características dos Ambientes das Medidas. 27

3.2. Descrição das medidas e da análise dos dados. 40

3.2.1. Equipamento para realização das medidas. $\quad 40$

3.2.2. Registro de mensagens protocolo NMEA. $\quad 40$

3.2.3. Descrição dos conteúdos das mensagens. 41

3.2.4. Posição do receptor GPS em cada medida. 42

3.2.5. Programa desenvolvido para análise em linguagem de programação Matlab.

3.3. Resultados das medidas. 44

3.3.1. Distribuições Cumulativas do Erro na Posição Horizontal. 45

3.3.2. Distribuições Cumulativas da Profundidade do Desvanecimento da Potência do Sinal Recebido. 49

3.3.3. Valores Médios e Desvios Padrões no erro na Posição Horizontal e Profundidade do Desvanecimento. 52

3.3.4. Histogramas do Número de Satélites vistos Simultaneamente 52

4. Análises de Resultados Numéricos das Simulações. 55

4.1. Introdução.

4.2. Modelos de Simulação. 56 
4.3. Ambientes urbanos utilizados na simulação.

4.3.1. Características dos tipos de ambientes para simulação.

4.3.2. Cálculos realizados para determinar a relação azimute- elevação para cada tipo de ambiente.

4.4. Resultados das simulações.

4.4.1. Resultado da Simulação do Ambiente 1. 73

a) Probabilidade de estados C e B baseado nos mapas e as posições $\begin{array}{ll}\text { dos satélites. } & 73\end{array}$

b) Valores do erro horizontal para diferentes porcentagens de tempo. $\quad 78$

c) Valores de desvanecimento para diferentes porcentagens de tempo. 84

4.4.2 Resultado da Simulação do Ambiente 2. 91

a) Probabilidade de estados C e B baseado nos mapas e as posições $\begin{array}{ll}\text { dos satélites. } & 91\end{array}$

b) Valores do erro horizontal para diferentes porcentagens de tempo. 96

c) Valores de desvanecimento para diferentes porcentagens de tempo. 103

4.4.3. Resultado da Simulação do Ambiente 3.

a) Probabilidade de estados C e B baseado nos mapas e as posições $\begin{array}{ll}\text { dos satélites. } & 111\end{array}$

b) Valores do erro horizontal para diferentes porcentagens de tempo. 114

c) Valores de desvanecimento para diferentes porcentagens de tempo. 119

$\begin{array}{lr}\text { 5. Conclusões. } & 125\end{array}$

Bibliografia. 


\section{Lista de figuras}

$\begin{array}{ll}\text { Figura 2.1- Constelação do sistema GPS } & 17\end{array}$

$\begin{array}{ll}\text { Figura 2.2- Segmentos do sistema GPS } & 18\end{array}$

Figura 2.3- Idéia básica de posicionamento do sistema GPS 20

Figura 2.4- Representação dos vetores posição do usuário e do satélite $\quad 22$

Figura 2.5- Relações de tempo para a pseudodistância 23

Figura 3.1- Lagoa Rodrigo de Freitas: ambiente das medidas 1 a $5 \quad 29$

Figura 3.2- FAETEC Ipanema: ambiente da medida $6 \quad 29$

Figura 3.3- Hotel Praia Ipanema: ambiente da medida $7 \quad 30$

Figura 3.4- Centro Tecnológico do Exército (CTEX): ambiente

da medida $8 \quad 30$

Figura 3.5- Lagoa Rodrigo de Freitas: local da medida 1

(árvore semiaberta) 31

Figura 3.6- Lagoa Rodrigo de Freitas: outra vista do local

da medida 1 (árvore semiaberta) 31

Figura 3.7- Lagoa Rodrigo de Freitas: local da medida 2

(árvore semifechada) 32

Figura 3.8- Lagoa Rodrigo de Freitas: outra vista do local

da medida 2 (árvore semifechada) 32

Figura 3.9- Lagoa Rodrigo de Freitas: local da medida 3

(árvore fechada)

Figura 3.10- Lagoa Rodrigo de Freitas: outra vista do local

da medida 3 (árvore fechada)

Figura 3.11- Lagoa Rodrigo de Freitas: local da medida 4

(árvore semifechada)

Figura 3.12- Lagoa Rodrigo de Freitas: outra vista do local

da medida 4 (árvore semifechada)

Figura 3.13- Lagoa Rodrigo de Freitas: local da medida 5

(céu claro) 
Figura 3.14- Lagoa Rodrigo de Freitas: outra vista do local

da medida 5 (céu claro) 35

Figura 3.15-FAETEC Ipanema: local da medida 6 (céu claro) 36

Figura 3.16- FAETEC Ipanema: outra vista do local

da medida 6 (árvore céu claro) 36

Figura 3.17- FAETEC Ipanema: outra vista do local da medida 6

$\begin{array}{ll}\text { (árvore céu claro) } & 37\end{array}$

Figura 3.18- Hotel Praia Ipanema: local da medida 7 (céu claro) 37

Figura 3.19- Hotel Praia Ipanema: outra vista do local da medida 7

(céu claro) 38

Figura 3.20- CTEx: local da medida 8 (árvore semifechada) 38

Figura 3.21- CTEx: outra vista do local da medida 8

(árvore semifechada)

Figura 3.22- CTEx: outra vista do local da medida 8

(árvore semifechada)

Figura 3.23- Distribuições cumulativas do erro horizontal

para as 8 medidas

Figura 3.24- Distribuições cumulativas do desvanecimento

para as 8 medidas

Figura 3.25- Histogramas correspondentes ao número

de satélites vistos simultaneamente para cada medida.

Figura 4.1- Distribuições de Probabilidade Rice (C),

Loo (S) e Suzuki (B)

58

$\begin{array}{ll}\text { Figura 4.2- Tipo de ambiente } 1 & 60\end{array}$

Figura 4.3- Tipo de ambiente $2 \quad 60$

Figura 4.4- Tipo de ambiente $3 \quad 60$

Figura 4.5- Variáveis utilizadas para o cálculo da relação

azimute-elevação de modo geral 62

Figura 4.6- Vista em 2D do azimute da rua e azimute do obsevador 63

Figura 4.7- Gráfico da relação azimute-elevação para o valores de $90^{\circ}$

para o azimute e de 1,0 para o fator w/h da rua 1 do ambiente 1

Figura 4.8- Gráfico da relação azimute-elevação para o valores de $90^{\circ}$

para o azimute e de 1,0 para o fator w/h da rua 1 do ambiente 2 
Figura 4.9- Gráfico da relação azimute-elevação para o valores de $90^{\circ}$

para o azimute e de 1,0 para o fator $\mathrm{w} / \mathrm{h}$ da rua 1 do ambiente 3

Figura 4.10- Probabilidade do estado "C" baseadas somente

nos mapas de estado para o ambiente 1

Figura 4.11- Probabilidade do estado "C" considerando tanto os mapas

de estado para o ambiente 1 quanto as posições dos satélites.

Figura 4.12- Valores do erro horizontal (m) não excedido durante

67 \% do tempo para o ambiente 1 em função do azimute da rua 1 (graus)

e do fator $\mathrm{h} / \mathrm{w}$.

Figura 4.13- Valores do erro horizontal (m) não excedido durante

$95 \%$ do tempo para o ambiente 1 em função do azimute da rua 1 (graus)

e do fator $\mathrm{h} / \mathrm{w}$.

Figura 4.14- Valores do desvanecimento (dB) não excedido durante

67 \% do tempo para o ambiente 1 em função do azimute da rua 1 (graus)

e do fator $\mathrm{h} / \mathrm{w}$.

88

Figura 4.15- Valores do desvanecimento (dB) não excedido durante

95 \% do tempo para o ambiente 1 em função do azimute da rua 1 (graus)

e do fator $\mathrm{h} / \mathrm{w}$.

Figura 4.16- Probabilidade do estado "C" baseadas somente

nos mapas de estado para o ambiente 2

Figura 4.17- Probabilidade do estado "C" considerando tanto os mapas

de estado para o ambiente 2 quanto as posições dos satélites.

Figura 4.18- Valores do erro horizontal (m) não excedido durante

$67 \%$ do tempo para o ambiente 2 em função do azimute da rua 1 (graus)

e do fator $\mathrm{h} / \mathrm{w}$.

Figura 4.19- Valores do erro horizontal (m) não excedido durante

$95 \%$ do tempo para o ambiente 2 em função do azimute da rua 1 (graus)

e do fator $\mathrm{h} / \mathrm{w}$.

Figura 4.20- Valores do desvanecimento (dB) não excedido durante

$67 \%$ do tempo para o ambiente 2 em função do azimute da rua 1 (graus)

e do fator $\mathrm{h} / \mathrm{w}$. 
Figura 4.21- Valores do desvanecimento (dB) não excedido durante 95 \% do tempo para o ambiente 2 em função do azimute da rua 1 (graus) e do fator $\mathrm{h} / \mathrm{w}$.

Figura 4.22- Probabilidade do estado "C” baseadas somente nos mapas de estado para o ambiente 3

Figura 4.23- Probabilidade do estado “C” considerando tanto os mapas de estado para o ambiente 3 quanto as posições dos satélites. Figura 4.24- Valores do erro horizontal (m) não excedido durante 67 \% do tempo para o ambiente 3 em função do azimute da rua 1 (graus) e do fator $\mathrm{h} / \mathrm{w}$.

Figura 4.25- Valores do erro horizontal (m) não excedido durante 95 \% do tempo para o ambiente 3 em função do azimute da rua 1 (graus) e do fator $\mathrm{h} / \mathrm{w}$.

Figura 4.26- Valores do desvanecimento (dB) não excedido durante 67 \% do tempo para o ambiente 3 em função do azimute da rua 1 (graus) e do fator $\mathrm{h} / \mathrm{w}$.

Figura 4.27- Valores do desvanecimento (dB) não excedido durante $95 \%$ do tempo para o ambiente 3 em função do azimute da rua 1 (graus) e do fator $\mathrm{h} / \mathrm{w}$. 


\section{Lista de tabelas}

Tabela 3.1-Nomes, localizações e principais características dos ambientes de medições $\quad 28$

Tabela 3.2- Posição do receptor GPS em cada medida 42

Tabela 3.3- Valores do erro na posição horizontal não excedidos durante 67\% e 95\% do tempo em cada local de medida 46

Tabela 3.4- Valores de desvanecimento não excedidos durante $67 \%$ e 95\% do tempo em cada local de medida

Tabela 3.5- Valores médios e desvios padrões do erro na posição horizontal e do desvanecimento.

Tabela 4.1- Características dos tipos de ambiente. $\quad 61$

Tabela 4.2- Probabilidades do estado “C” baseadas somente nos mapas de estado para o ambiente 1

Tabela 4.3- Probabilidades do estado “C” baseadas nos mapas de estado para o ambiente 1 e nas posições dos satélites.

Tabela 4.4- Valores do erro Horizontal (m) não excedido durante $67 \%$ do tempo para o ambiente 1

Tabela 4.5- Valores do erro Horizontal (m) não excedido durante 95\% do tempo para o ambiente 1

Tabela 4.6- Valores do desvanecimento (dB) não excedidos durante $67 \%$ do tempo para o ambiente 1

Tabela 4.7- Valores do desvanecimento (dB) não excedido durante 95\% do tempo para o ambiente 1

Tabela 4.8- Probabilidades do estado "C" baseadas somente nos mapas de estado para o ambiente 2

Tabela 4.9- Probabilidades do estado "C" baseadas nos mapas de estado para o ambiente 2 e nas posições dos satélites.

Tabela 4.10- Valores do erro Horizontal (m) não excedido durante $67 \%$ do tempo para o ambiente 2

Tabela 4.11- Valores do erro Horizontal (m) não excedido durante 95\% do tempo para o ambiente 2 
Tabela 4.12- Valores do desvanecimento (dB) não excedidos durante $67 \%$ do tempo para o ambiente 2

Tabela 4.13- Valores do desvanecimento (dB) não excedido durante 95\% do tempo para o ambiente 2

Tabela 4.14- Probabilidades do estado " $C$ ” baseadas somente nos mapas de estado para o ambiente 3

Tabela 4.15- Probabilidades do estado " $\mathrm{C}$ ” baseadas nos mapas

de estado para o ambiente 3 e nas posições dos satélites.

Tabela 4.16- Valores do erro Horizontal (m) não excedido durante $67 \%$ do tempo para o ambiente 3

Tabela 4.17- Valores do erro Horizontal (m) não excedido durante 95\% do tempo para o ambiente 3

Tabela 4.18- Valores do desvanecimento (dB) não excedidos durante $67 \%$ do tempo para o ambiente 3

Tabela 4.19- Valores do desvanecimento $(\mathrm{dB})$ não excedido durante 95\% do tempo para o ambiente 3 